\title{
Medium Access Control Layer Design and Simulation for 802.16 System
}

\author{
Yu Cheng, Hongwei Zhao, Yucui Li, Shihui Pei \\ College of Computer Science and Technology, Jilin University, Changchun, 130012, China \\ e-mail: unplug2007@163.com
}

\begin{abstract}
In order to analyze transmission performance of broad band wireless communication system based on 802.16 protocol, address the key issues of QoS scheduling algorithm, and network congestion, etc, we present our design of WiMAX medium access control (MAC) layer based on 802.16 protocol in this paper. The implemented design comprises the servicespecific convergence sub-layer (CS), the MAC common part sub-layer (CPS), a call admission control mechanism, and a scheduler. In the next simulation experiment, we tested the throughput and average delay of the system which we designed under the conditions in different time or number of clients. The design and simulation had provided a platform and foundation for further to improve the efficiency of bandwidth management algorithm, scheduling algorithm, QoS management, and enhancing the performance of WiMAX systems.
\end{abstract}

\section{Keywords- 802.16; MAC; Simulation experiments; CS;CPS}

\section{FOREWORD}

IEEE 802.16 is a kind of broadband wireless access (BWA) standard which focuses on wireless metropolitan area networks (WMANs). WiMAX is a concrete realization of 802.16 protocol [1]. It is important to design a efficacious MAC architecture, because IEEE 802.16 has not described the algorithm of QoS scheduling and net block control in detail [2]. We can solve this problem through simulating and analysis. NS-2 is very commonly used network simulation software in the industrial and academic field. However, the software is not designed to support IEEE 802.16, for this reason, in this paper we will design the MAC architecture based on 802.16 protocol and simulate on NS-2. The design will be developed in the way of inheriting the original NS-2 class.

\section{DESIGN OF MAC LAYER BASED ON 802.16}

Developed using object-oriented programming language $\mathrm{C}++$, The created WiMAX are based on the original NS-2 network components. Figure 1 shows a variety of object types and their relationship.

In the design, WiMAX is divided into service specific convergence sub-layer (CS), MAC common part sub-layer (CPS), and physical layer (PHY) [3]. design.

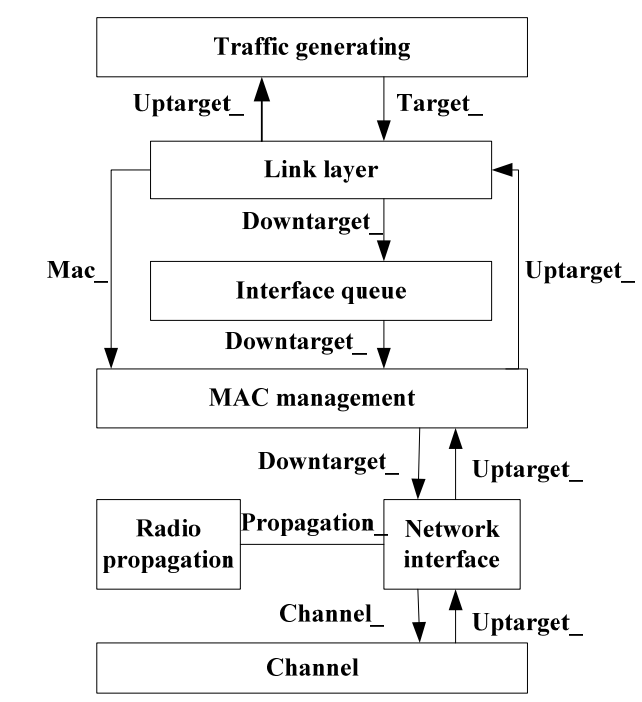

Figure 1. 802.16-based MAC layer and NS-2 diagram

\section{A. CS sub-layer design}

Service specific convergence sub-layer (CS) has two main functions: (1) Mapping IP which is received from up-layer into service flow identifier (SFID), or Mapping SFID into IP. (2) Receive SFID and transport CID (TCID) [5].

1) IP and SFID mapping: SDU is data package from uplayer. It contains the information about destination address, service type, etc. The IP-SFID mapping function should record the information, and classify the packet by different parameters, in order to prepare for IP-MAC mapping. One SFID will be used to confirm the QoS parameters in downlink (DL), or for IP address lookup in uplink (UL).

2) SFID and TCID mapping: SFID-TCID mapping is the main function of CS sub-layer. The function associates the service flow QoS class with connection.

For example, in UL, when the package header does not contain TCID, the subscriber station (SS) will send a bandwidth request header to the base station (BS) with a main CID in it, so the BandwidthRequest( ) function will be activated for data transmission requesting. SS add, change, or delete obtained bandwidth by sending bandwidth management messages as DSA, DSC, and DSD.

For DL, the decision if distribute a TICD to a SS or not will be decided by insert_SFID( ) function. If the decision is 
a no, the function will generate a no longer used TCID for $\quad$ SS, or send that MSDU to an appropriate queue.

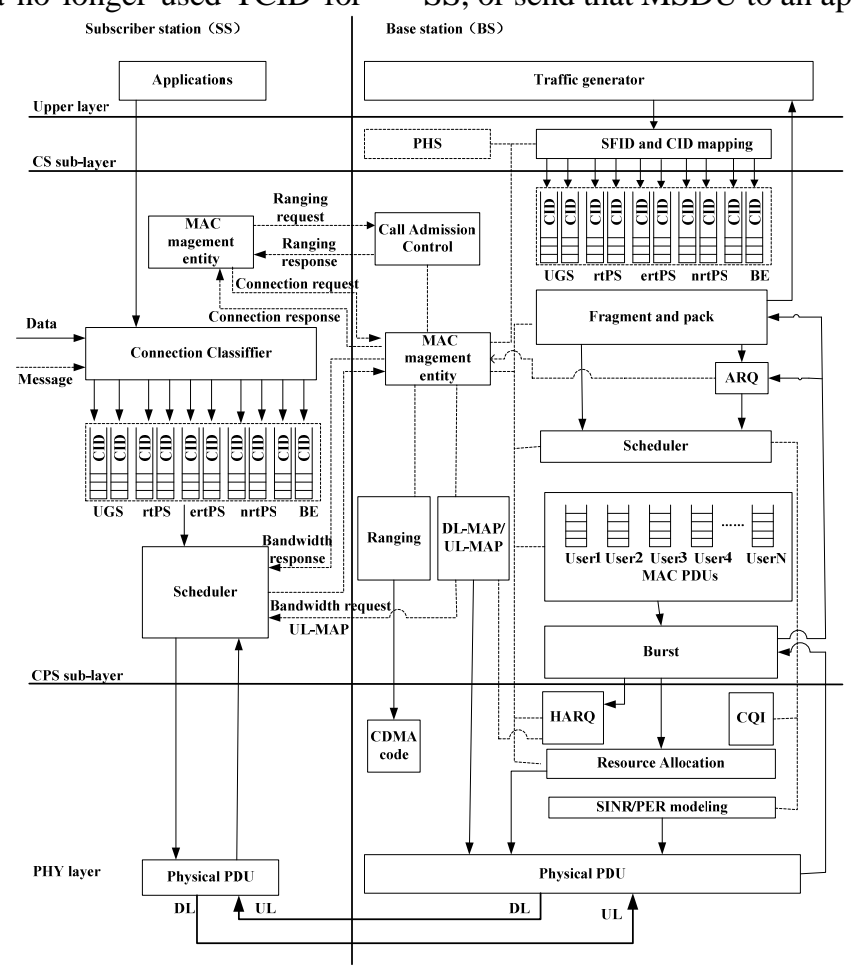

Figure 2. Tthe structure of the WiMAX system design

\section{B. CPS sub-layer design}

CPS sub-layer is the major part of MAC layer, which generate running and management message for MAC system [6], such as DCD, UCD, DSD, DSA, DSC, DL-MAP, ULMAP, RNG-REQ, RNG-RSP, etc.

1) Ranging function: When SS first access network, the first step is ranging function. The new SS should scan downlink channel, and establish synchronization with BS. After the process of establishing finished, the SS will obtain radio parameter by receiving UCD message. In this way, SS can confirm the channel information for data transmission. BS broadcast the UCD message to SS periodically. When an unregistered SS receives a packet from the queue object, ranging function will be started to informing BS that a SS is entering system. The SS sends REG-REQ management message to compete for system access rights in ranging gap. BS will confirm the request by modulating quadrature phase shift keying (QPSK) 1/2 coding rate. Access procedure follows random countdown mechanism. The start time of countdown between 0 and $C W_{\min }-1 . C W_{\min }$ express the size of the minimum contention window, it is equal to 32 .

2) Medium access control management and call admission control (CAC) mechanism: MAC management includes five kinds of messages, namely UL-MAP, DL-MAP, Band width request (BR), UCD and DCD. Each of them has its own management of message types, and they can distinguish themselves from each other. DCD message includes the management of the message types are DL channel ID, TLV overall channel encoding information, and DL burst profile. DCD channel encoding is composed by TLV which contains all of channel information, such as downlink bust profile, physical type, frame duration, number of channel, number of frame, duration code of frame, BISD, the DL frequency, receive/transmit transition gap (RTG), transmit/receive transition gap (TTG), and power adjustment rule. DCD focus on DL burst profile. It consists of DIUC (mapping DL-MAP) and TLV encoding information.

UCD message contains some message management types, the start of ranging countdown, the end of ranging countdown, TLV overall channel encoding information, countdown start request, as well as countdown end request. The major part of UCD is TLV overall channel encoding information, which has constituted the UL burst profile. UL burst profile is the same of including FEC encoding type and modulation type as the DL burst profile.

The traffic generating agent has generated all of the traffic flow. These flows will be dealt into basic packet object. The packets will pass through the interface queue and arrive in the MAC module. They will become the MSDU in MAC module. After that, the system will active the Mapping( ) function of SFID for assorting the MSDU with some groups, like UGS, rtPS, ertPS, nrtPS, and BE. MSDU will be mapped into the queue labeled like SFID number when SFID is active, or else, a TCID will be distributed by Mapping( ) function of SFID, and the relevant SFID will be activated by the function too. 
The MSDU will be mapped into relevant queue and ready to be served after the service flow is classified. During the procedure, the BS should refers to the BWMT( ) function for band width management whether in uplink channel or in downlink channel. The BWMT( ) function takes the call admission control (CAC) algorithm, in the process of running, CAC algorithm follows the principle of first in first serve (FIFS) to deal with the bandwidth request. If the bandwidth is enough, the request will be accepted, otherwise, it will be refused by CAC.

3) Priority queue algorithm: The packets receive form upper layer will be sent to Priority( ) function firstly, either in BS or SS. The function will classify the service types with assigning different priority such as UGS(5), rtPS(4), ertPS(3), nrtPS(2), and BE(1). The Priority( ) will create a dedicated queue in accordance SFID for planting these packets. The packets will be treated into some MSDUs and subsection into different queue of function such as ertPS_Q( ), nrtPS_Q( ), BE_Q( ), etc, finally.

4) Schedule algorithm: The Schduler( ) function take charge of choosing MSDU from different queue base on the bandwidth request which has been accept [7]. The schedule algorithm that we designed is the weighted Round-Robin algorithm. First of all, there are five kinds of service types, including UGS, rtPS, ertPS, nrtPS, and BE [8]. In downlink, we express the five service types in different variable as $q 5$, q4, q3, q2, and q1. Then we assign a value which is less than 1 to each variable.

In the first round, the serving quantity that is assigned to each classification base on the following formula:

$$
\begin{aligned}
& B_{\text {type }}^{T}=\min \left(R_{\text {type }}, B_{\text {total }} * q i\right), i \in\{1, \ldots, 5\} \\
& \sum_{i=1}^{5} q i \leq 1
\end{aligned}
$$

Rtype indicates the total number of the service types which request the bandwidth. Btotal indicates the total number of the bandwidth that system can afford.

$\{q 5, q 4, q 3, q 2, q 1\}$ are variables which can be set according to system need.

In the second round, the Scheduler( ) function will serve the remaining request which has not been served in accordance with the order of priority. If all of the request in priority i have been served, the Scheduler( ) function will keep serving the requests in priority $\mathrm{i}+1$. The procedure will be repeated again and again, until all of the bandwidth resource is consumed or there is no un-served request.

This algorithm is able to ensure that the BE service type can be served by minimum bandwidth, even in the moment that the traffic load is the heaviest.

5) Uplink map and downlink map: Uplink map (ULMAP) and downlink map (DL-MAP) announce the allocation of UL and DL by being created periodically. The two messages are generated by ULmap-Handler( ) and DLmapHandler( ) respectively.

6) Fragmentation/De-fragmentation

and Packing/Unpacking: The fragmentation and packing of a packet is completed by PDU_Generator( ) function. The function grasps MSDU packets form QoS queue, such as UGS, rtPS, ertPS, nrtPS, BE. Then treat MPDU base on Scheduler( ). The function generates a normal MAC header for each payload of data. The fragmentation is a procedure of dividing a MSDU into one or more MSDU.

After a group of data has been fragmented or packed, the Transmit_Data will be active by scheduler for data transmission.

The Assembler( ) function is used to de-fragment or unpack data.

7) The timer class of 802.16: The timer class of 802.16 inherits three important functions from Handler class. The start( ) function performs the function of triggering time to start. If the event happens before the time is up, the stop( ) function will be active to stop the time. The Handle( ) function performs the function of triggering time-out event.

In WiMAX system, the timer class constraints the progress of the incident between the BS and the SS.

There is another kind of timer performs function of backoff the given specific time before the burst event happens or the expected time expires.

\section{SIMULATION EXPERIMENT AND PERFORMANCE TEST}

The specific parameters of simulation experiment are shown in Table I and Table II. We set one BS serve for ten SSs at the same time. The coverage of the BS is $1000 \mathrm{~m}$ $1000 \mathrm{~m}$. All of the SSs distributed randomly round the BS.

The system transmission rate decides on the distance between the SS and BS, and the modulation versus distance model is based on system transmission rate.Each of SS adopt QPSK 1/2 encoding rate for generating the ranging request.

We have designed three varieties of service flow, namely, UGS, nrtPS and BE. All of the traffic flows are generated by traffic generating agent. The TCP/IP traffic is treated into DL transmission from BS to SS. On the other hand, the UL transmission is traffic from SS to internet. There are 14 groups of UGS connection totally, for CBR traffic, each connection occupies a fixed 1024kbps data channel.There are 4 groups of nrtPS connection totally, for VBR traffic, each connection occupies averagely 448kbps data rate. Distributes averagely data length between 200bytes-980bytes by setting Uniform(200,980), and time interval by Uniform($0.5,0.5)$. Namely each of the connection occupies $1.5 \mathrm{Mbps}$. $\mathrm{BE}$ and VBR are similar in traffic model, but the group of connection is 2. The data length of BE between 512bytes1024bytes. The order of traffic priority are UGS, nrtPS, and $\mathrm{BE}$. Scheduler is weighted Round-Robin mode.

TABLE I. PARAMETERS OF SIMULATION IN MAC LAYER

\begin{tabular}{ll}
\hline Parameters & value \\
\hline Basic CID & $1-1000$ \\
Primary CID & $1001-2000$ \\
Broadcast CID & 65535 \\
Initial ranging CID & 0 \\
Transport/secondary Mgt. CIDs & $2001-65278$ \\
DL/UL ratio & $2: 1$ \\
\hline
\end{tabular}


Max. number of bandwidth req. retry 10

Max. number of ranging retry 10

Bandwidth request opp. per frame 12 OFDMA symbols

Ranging opp. per Frame 12 OFDMA symbols

CWmax 1024 opps

CWmin 32 opps

No. of subchannels $\quad 30$

No. of OFDMA symbol per frame $\quad 49$

No. of OFDMA symbol per frame 48(data portion)

SFID range 1-4294967295

TABLE II. TIME PARAMETERS OF SIMULATION

\begin{tabular}{ll}
\hline Parameters & value \\
\hline RTG & $200 \mu \mathrm{s}$ \\
TTG & $200 \mu \mathrm{s}$ \\
Bandwidth request interval & $1200 \mu \mathrm{s}$ \\
Ranging interval & $1200 \mu \mathrm{s}$ \\
OFDMA frame length & $5 \mathrm{~ms}$ \\
OFDMA symbol time & $100 \mu \mathrm{s}$ \\
DCD/UCD period & $10 \mathrm{sec}$ \\
T1-T26 & as defined in IEEE 802.16 \\
\hline
\end{tabular}

The time of simulation sets for 50 seconds, against this time, the transmission generating throughput uninterrupted. The throughput has been rising versus time. The reason of the low throughput at the beginning of simulation is that SS need lots of time to deal with ranging and bandwidth request in this period. The throughput will reach around $2.8 \mathrm{Mbps}$ finally. The curve of that track of throughput transforms versus time in simulation is shown in Fig.3.

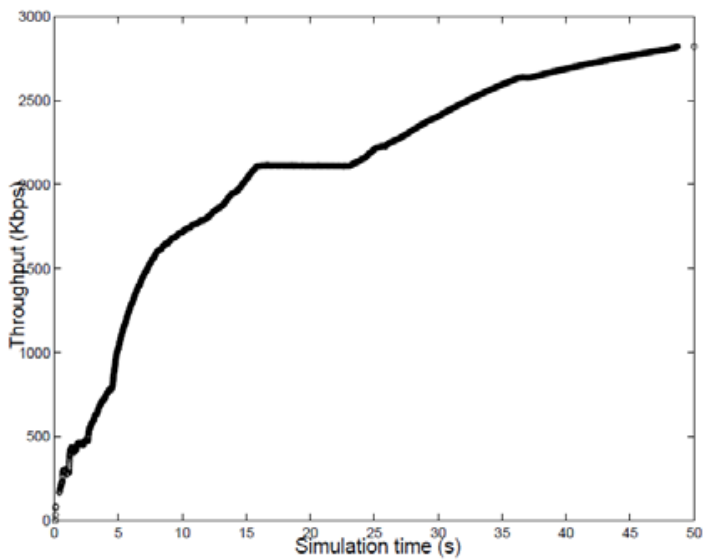

Figure 3. The throughput versus 50 second simulation time

The system average delay changes with the number of SS, as shown in Fig.4. We can see that average delay get longer with the number of base stations increasing. This is the result of clashing between the initial ranging and bandwidth request. When bandwidth request process finished, system delay will be balanced because BS assigns the traffic time for SS. For the spectral resource is limited, the efficiency of scheduling for different types of traffic service will be the key factor to the system delay.

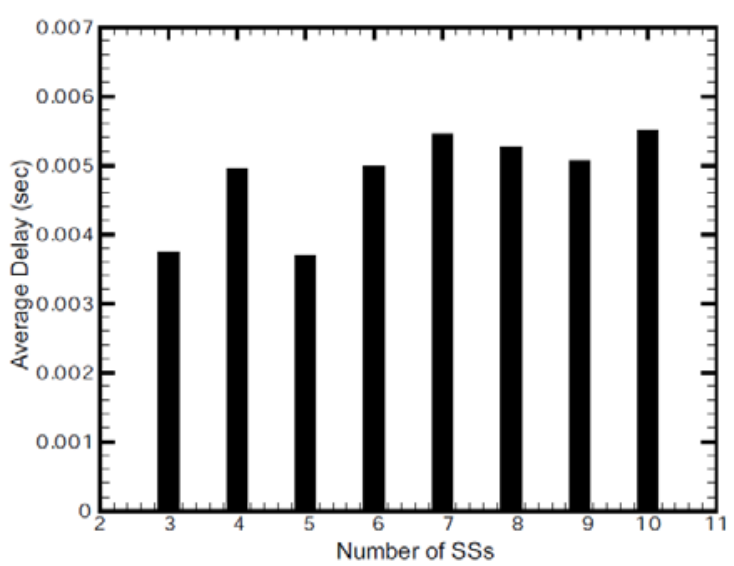

Figure 4. the average system delay versus different SS number

\section{CONCLUSIONS}

This paper designs and implements MAC layer of WiMAX system on NS-2 platform. The designed MAC layer is based on IEEE 802.16 standard. Implement the basic function of WiMAX in PMP mode [4], and bandwidth management, scheduling for different traffic service types. Test the throughput versus different simulation time and the average delay versus different number of SSs.

\section{REFERENCES}

[1] IEEE 802.16 Working Group, "IEEE Standard for Local and Metropolitan Area Networks-Part 16: Air Interface for Fixed Broadband Wireless Access Systems,” IEEE Std. 802.16-2004, October 2004.

[2] -, "Part 16: Air Interface for Fixed Broadband Wireless Access Systems-Amendment 2: Medium Access Control Modifications and Additional Physical Layer Specifications for 2-11 GHz," IEEE Std. 802.16a, Apr. 2003.

[3] -, "Part 16: Air Interface for Fixed Broadband Wireless Access Systems-Amendment 2: Physical and Medium Access Control Layers for Combined Fixed and Mobile Operation in Licensed Bands,” IEEE Std. 802.16e, Dec. 2005.

[4] Simon Redana, Matthias Lott, Performance Analysis of IEEE 802.16a in Mesh Mode [C], International Conference on Computer Science and Informatics, 2005.

[5] C. Eklund et al., "IEEE Standard 802.16: A Technical Overview of the WirelessMAN Air Interface for Broadband Wireless Access," IEEE Commun. Mag., 40(6):98-107, June 2002.

[6] Gupta, P.; Kumar, P. R. the capacity of wireless networks [J]. Information Theory, IEEE Transactions on Volume 46, Issue 2, March 2000 Page: 388-404.

[7] Min Cao, Wenchao Ma, Xiaodong Wang, etc. Modelling and Performance Analysis of the Distributed Scheduler [C]. ACM MobiHoc' 05, pp. 78-79, 2005

[8] Chengzhi Li; Knightly, E, W.. Coordinated network scheduling: a framework for end-to end services [J]. Network Protocols, 2000. Proceedings. 2000 International Conference on, 14-17 Nov. 2000, Pages:69-7. 